\title{
Calibration of the high-doping induced ballistic band-tails tunneling current with In0.53Ga0.47As Esaki diodes
}

\author{
Jasper Bizindavyi ${ }^{1,2}$, Anne S. Verhulst ${ }^{1}$, Quentin Smets ${ }^{1}$, Devin Verreck ${ }^{1}$, \\ Nadine Collaert ${ }^{1}$, Anda Mocuta ${ }^{1}$, Bart Soré ${ }^{1,2,3}$ and Guido Groeseneken ${ }^{1,2}$ \\ ${ }^{1}$ imec, 3001 Leuven, Belgium, Kapeldreef 75, Email: jasper.bizindavyi@imec.be; ${ }^{2}$ Department of Electrical \\ Engineering, KU Leuven, 3001 Leuven, Belgium; ${ }^{3}$ Department of Physics, UAntwerp, 2020 Antwerp, Belgium
}

\section{INTRODUCTION}

The growing demand for power efficient devices has accelerated the research into the use of the tunnel field-effect transistor (TFET) in future ultra-low power applications because of its promising potential for sub-60 mV/dec subthreshold swing achieved through quantum mechanical band-to-band tunneling (BTBT) [1]-[3]. Unfortunately, a significant gap between theoretical predictions and experiments remains to be bridged [2]. Considerable efforts are being made to develop models for some of the main causes of suboptimal TFET performance such as trap-assisted tunneling (TAT) [4], [5], phonon-assisted tunneling (PAT) [6], and Auger generated leakage currents [7]. However, aside from qualitative analyses [8] and purely predictive work on the device impact of tunneling transitions involving high-doping induced band-tails states in InSb nanowire TFETs [9] and 2D-TFETs [10], no attempts have been made to calibrate these contributions. This work aims to fill this gap by developing and calibrating an approximate ballistic semi-classical (SC) model for high-doping induced band-tails using the experimental I-V data of $\operatorname{In}_{0.53} \mathrm{Ga}_{0.47} \mathrm{As} p-i-n$ Esaki diodes [11]. The hypothesis is posited that the mismatch between measurement and simulation in the negative differential resistance regime (see Fig. 1), which cannot be explained by SC TAT models, is caused by ballistic bandtails tunneling. The calibration thus gives an upper limit to the band-tails current. Lastly, the impact of band-tails on the performance of a $p-n-i-n$ TFET is investigated.

\section{SEMI-CLASSICAL BAND-TAILS MODEL}

To obtain large $I_{O N}$ and steep-onset transfer characteristics, a TFET is often highly doped to achieve short tunnel paths at the tunnel junction [1]. However, large doping concentrations deform the band structure of a material [12], [13]. This results in both a doping-induced bandgap narrowing (BGN) [13], [14] and the appearance of band-tails in the density of states (DOS) of the conduction and valence band [12], [15]. We developed an approximate model that can be used to calibrate the resulting band-tails tunneling current, using an approach similar to [9] for the band-tails description but now for implementation in a SC solver. In particular, an artificial energy dispersion relation $E_{b t s, c / v}(k)$, where $E_{b t s}$ is the band-tails state energy and $k$ is the wave vector magnitude, of the following form is assumed:

$$
k=k_{1, c / v} \exp \left(\frac{ \pm\left(E_{b t s}-E_{1, c / v}\right)}{3 E_{0, c / v}}\right) \text { for: }\left\{\begin{array} { l } 
{ E < E _ { 1 , c } } \\
{ E > E _ { 1 , v } }
\end{array} \text { or } \quad E _ { b t s } = E _ { 1 , c / v } \pm 3 E _ { 0 , c / v } \operatorname { l n } ( \frac { k } { k _ { 1 , c / v } } ) \text { for: } \left\{\begin{array}{l}
k<k_{1, c} \\
k<k_{1, v}
\end{array}\right.\right.
$$

where $+(-)$ refers to the conduction(valence) band-tails and where, $k_{1, c / v}$ and $E_{1, c / v}$ specify the starting point of the conduction/valence band-tails, and $E_{0, c / v}$ determines the slope of the dispersion relation, see Fig. 2. Note that the energy dispersion relation given by Eq. (1) implicitly assumes that there exists a one-to-one correspondence between the energy of a band-tails state and a wave vector $k$, which is only the case for delocalized states such as bulk valence and conduction band states and the band-tails states close to the conventional band edges [16].

From the expression for $E_{b t s, c / v}(k)$ in Eq. (1), an expression for the band-tails density-of-states can be found:

$$
g_{b t s, c}\left(E_{b t s}\right)=\frac{k_{1, c / v}^{3}}{3 \pi^{2} E_{0, c / v}} \exp \left(\frac{ \pm\left(E_{b t s}-E_{1, c / v}\right)}{E_{0, c / v}}\right) .
$$

From Fig. 3, it is clear that this model predicts an exponential energy dependence of the band-tails DOS. This is in qualitative agreement with both theoretical predictions [17] and experimental observations [18].

\section{BAND-TAILS TUNNELING CURRENT}

We have employed our SC band-tails model to calibrate the parasitic band-tails tunneling current. Because band-tails states close to the conventional band edges can be considered to be spatially extended states [16], we treat the tunneling between valence and conduction band-tails states similarly to conventional band-to-band tunneling. More specifically, the requirement is imposed that the energy and the wave vector components orthogonal to the transport direction, $k_{\perp}$, are conserved during the tunneling transition. This requirement allows for a first exploration of the impact of bandtails. The following expression, derived from Tsu-Esaki [19], is then used for the ballistic band-tails tunneling current:

$$
J_{b t s \leftrightarrow b t s}=\frac{q}{2 \pi^{2} \hbar} \int_{x_{L}}^{x_{R}} q \vec{E}(x) \int_{\forall k_{\perp}} k_{\perp} T\left(E_{b t s, v}(x), k_{\perp}\right)\left[f_{n}\left(E_{b t s, c}\left(x^{\prime}, k_{\perp}\right)\right)-f_{p}\left(E_{b t s, v}\left(x, k_{\perp}\right)\right)\right] \mathrm{d} k_{\perp} \mathrm{d} x
$$


with $\vec{E}(x)$ the local electric field, $x_{L / R}$ the position of the left/right contact, $T$ the tunneling probability which for conventional BTBT is given by $T_{W K B}=\exp \left(-2 \int \kappa_{\mathrm{x}}(x) \mathrm{d} x\right), x^{\prime}=x+l_{t u n}$ and $f_{n(p)}$ the electron (hole) Fermi-Dirac distribution function. The attenuation experienced by an electron that tunnels from one band-tails state to another is then determined by taking the contour integral of the imaginary wave vector value $\kappa_{x}$ along the followed tunnel-path. We assume that the path taken by the electron bridges the effective bandgap between the valence and conduction band-tails state, as illustrated in Fig. 4. Therefore, in Eq. (3), we have used the WKB approximation in the limit of a uniform electric field for the transmission probability $T$ [20]:

$$
T\left(E(x), k_{\perp}\right)=\exp \left(\frac{-\pi E_{G, b t s, a v g}^{2}\left(x, k_{\perp}\right) m_{e 0}}{4 \hbar p_{c v} q\left|\vec{E}_{a v g}\right|}\right)
$$

with $E_{G, b t s, a v g}\left(x, k_{\perp}\right)=\operatorname{avg}\left(E_{b t s, c}\left(k_{\perp}\right)-E_{b t s, v}\left(k_{\perp}\right)\right)$ the average effective bandgap over the tunnel-path, $\left|\vec{E}_{\text {avg }}\right|$ the equivalent uniform electric field over the tunnel-path and with $p_{c v}$ the interband momentum matrix element coupling the valence and conduction band, found by Kane to be: $p_{c v}=m_{e 0} / 2 \sqrt{E_{G} / m_{r}}$, with $m_{r}$ the reduced tunneling mass. Note that using this expression for $p_{c v}$ is equivalent to saying that the coupling between valence and conduction bandtails states is described in the same way as the coupling between conventional valence and conduction band states. Unlike band-tails states close to the conventional band edges, band-tails states deep in the bandgap are very localized in space which decreases the efficiency of ballistic tunneling transitions involving these states. We have implemented this effect with an abrupt transition whereby only the tunneling transitions involving band-tails states for which $E_{b t s, v} \leq E_{V}+E_{\text {edge, } v} \& E_{b t s, c} \geq E_{C}-E_{\text {edge,c}}$ contribute to $J_{b t s \leftrightarrow b t s} . E_{\text {edge }, c / v}$ is typically taken equal to $100 \mathrm{meV}$. To implement tunneling between conventional band states and band-tails states, the requirement that the tunneling transition conserves the energy and orthogonal wave vector components is also imposed. As a result, expressions similar to Eq. (3) were found for the tunneling current between the conventional valence band and conduction bandtails, $J_{v \leftrightarrow c, b t s}$, and the tunneling current between the conventional conduction band and valence band-tails, $J_{c \leftrightarrow v, b t s}$.

\section{CALIBRATION OF BAND-TAILS MODEL AND IMPACT ON TUNNEL-FET PERFORMANCE}

The SC band-tails model has been calibrated using Esaki diodes with an intrinsic region of 18nm, as detailed in [11]. Using Fig. 1, one can compare the experimental current density (black curve) with the simulated conventional BTBT (red curve, calculated using the dynamic nonlocal BTBT model in Sentaurus Device [21] after optimization of the Fermi level position, the BGN, and the effective masses used in the BTBT model, where the heavy hole mass was used as transverse mass [22]). It is clear that, unlike in reverse-bias, there remains a significant mismatch in forwardbias between $-0.3 \mathrm{~V}$ and $0 \mathrm{~V}$. Recently, it has been shown that this mismatch can never completely be removed [22]. The SC band-tails model's parameters are calibrated based on the hypothesis that the band-tails are entirely responsible for the forward-bias mismatch in the $p-i-n$ Esaki diodes. At the interface between a highly doped region and the intrinsic region, the DOS and the energetic extension of the band-tails into the bandgap are reduced such that after a distance of $3.5 \mathrm{~nm}$ into the intrinsic region both the conduction and valence band-tails have disappeared. This results in a decrease of the band-tails DOS proportional to that of the doping profile, which decays with $6 \mathrm{~nm} / \mathrm{dec}$ [11]. However, the calibration is found to be rather insensitive to the exact length of the transition region, as the calibration result for the case of a $2 \mathrm{~nm}$ transition region is found to be identical to that for a $3.5 \mathrm{~nm}$ transition region.

The calibration result, see Fig. 5, shows that a complete match over the entire voltage range can be achieved when both the BTBT and the band-tails tunneling contributions are taken into account. Table I gives the calibrated values of the model parameters. Note that we are still able to obtain a satisfactory match for values of $E_{\text {edge,c/v }}$ as low as $50 \mathrm{meV}$ if the values for $E_{0, c / v}$ are increased accordingly up to a value of about $100 \mathrm{meV}$. At this point, we remark that the obtained match with our model should not be used as proof that PAT, thermal band-tails or PAT involving highdoping induced band-tails states, do not contribute to the forward-bias mismatch. The obtained calibration represents the highest possible contribution to the current because of ballistic high-doping induced band-tails tunneling.

As a first application, the now calibrated band-tails model has been applied to an $\operatorname{In}_{0.53} \mathrm{Ga}_{0.47} \mathrm{As} p$ - $n$-i-n double-gate TFET structure, see Fig. 6. To compensate for the higher doping concentrations in the TFET compared to the Esaki diode, a factor of $\sqrt[3]{2.5}$ has been added to the calibrated values of $k_{1, c / v}$ to increase the band-tails DOS. We have thus assumed that $g_{b t s, c / v} \propto N_{\text {doping }}$. The simulation results, Fig. 7, show that the band-tails tunneling contributions dom-

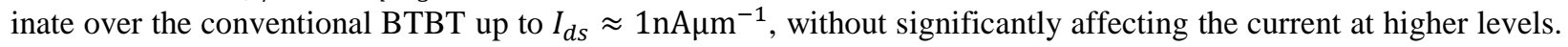
The band-tails contributions have no impact on the TFET performance, when a value of $50 \mathrm{meV}$ for $E_{\text {edge,c/v}}$ is used.

\section{CONCLUSION}

A SC model for high-doping induced band-tails and the corresponding ballistic tunneling contributions has been developed by assuming an artificial energy dispersion relation that correctly reproduces the exponential energy 
dependence of the band-tails DOS. The model parameters have been calibrated using a highly doped $\operatorname{In}_{0.53} \mathrm{Ga}_{0.47} \mathrm{As}$ $p$-i-n Esaki diode, resulting in an Urbach energy, $E_{0, c / v}$, of $70 \mathrm{meV}$. As a first application, we have shown the performance impact on a $p-n-i-n$ TFET, predicting dominant band-tails contributions for current levels $\leq 1 \mathrm{nA} \mu \mathrm{m}^{-1}$, which calls for an additional and more detailed investigation of the impact of band-tails on different TFET architectures. Future work will focus on refining the SC model and applying the calibration procedure to different temperatures, doping levels and materials.

\section{ACKNOWLEDGMENT}

J. Bizindavyi gratefully acknowledges FWO-Vlaanderen for a Strategic Basic Research PhD fellowship. This work was supported by imec's Industrial Affiliation Program.

\section{REFERENCES}

[1] D. Verreck et al., in Wiley Encyclopedia of Electrical and Electronics [12] S. C. Jain et al., Adv. Electron. Electron Phys., vol. 82, no. C, 1991. Engineering. Hoboken, NJ, USA: John Wiley \& Sons, Inc., sep 2016. [13] P. Van Mieghem, Ph.D. dissertation, KU Leuven, 1991.

[2] H. Lu and A. Seabaugh, IEEE J. Electron Devices Soc., vol. 2, no. 4, jul 2014. [14] K.-F. Berggren and B. Sernelius, Solid-State Electron., vol. 28(1-2), jan 1985.

[3] A. M. Ionescu et al., Nature, vol. 479, no. 7373, pp. 329-337, 2011. [15] P. Van Mieghem, Rev. Mod. Phys., vol. 64, no. 3, pp. 755-793, jul 1992.

[4] S. Sant et al., IEEE Trans. Electron Devices, vol. 63, no. 11, nov 2016.

[5] M. Mohammed et al., J. Appl. Phys., vol. 120, no. 24, p. 245704, 2016.

[6] J. Charles et al., J. Comput. Electron., vol. 15, no. 4, dec 2016.

[7] J. Teherani et al., J. Appl. Phys., vol. 120, no. 8, p. 084507, 2016

[8] S. Agarwal and E. Yablonovitch, IEEE Trans.Electron Devices, vol. 61, 2014.

[9] M. A. Khayer and R. K. Lake, J. Appl. Phys., vol. 110, no. 7, p. 074508, 2011.

[10] H. Zhang et al., in 2016 IEEE IEDM, dec 2016, pp. 30.3.1-30.3.4.

[11] Q. Smets et al., J. Appl. Phys., vol. 115, no. 18, p. 184503, may 2014.

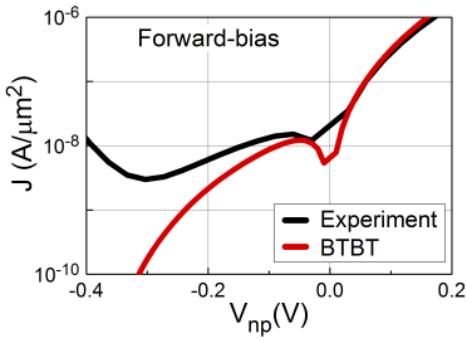

Fig. 1. Remaining discrepancy between experiment and simulation in forward-bias.

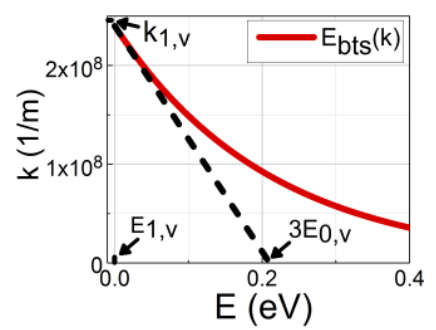

Fig. 2. Energy dispersion relation of the valence band-tails states. $E_{0, v}, k_{1, v}$ and $E_{1, v}$ are given in Table I.

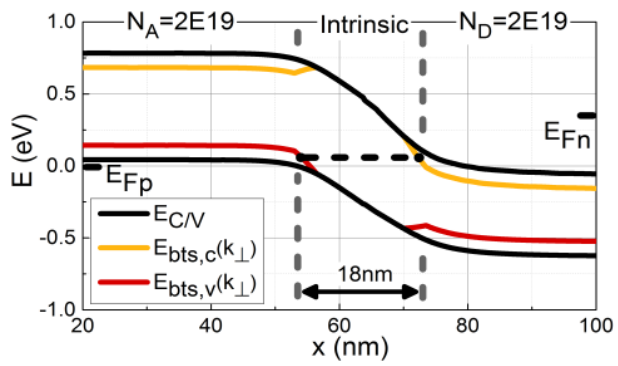

Fig. 4. The followed tunnel-path (black dashed) between a valence and conduction band-tails state bridges the bandgap between these band-tails states.

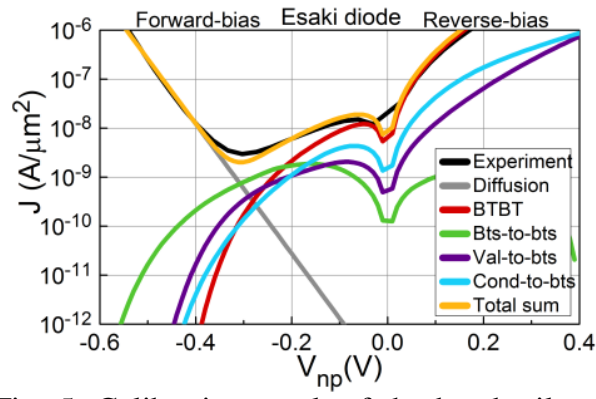

Fig. 5. Calibration result of the band-tails model using the experimental data of [11]. The calibration results for $E_{0, c / v}, k_{1, c / v}$ and $E_{1, c / v}$ and values for $E_{e d g e, c / v}$ are given in Table I.
[16] J. Dong and D. A. Drabold, Phys. Rev. B, vol. 54, no. 15, 1996.

[17] J. Serre et al., Phys. Rev. B, vol. 23, no. 4, pp. 1971-1976, 1981.

[18] J. I. Pankove, Phys. Rev., vol. 140, no. 6A, pp. A 2059 - A 2065, 1965.

[19] R. Tsu and L. Esaki, Appl. Phys. Lett., vol. 22, no. 11, pp. 562-564, jun 1973.

E. O. Kane, J. Appl. Phys., vol. 32, no. 1, p. 83, 1961.

[21] SYNOPSYS, "SDevice user guide," Ver. K-2015.06, 2015.

[22] J. Bizindavyi, Master thesis, KU Leuven, 2016.

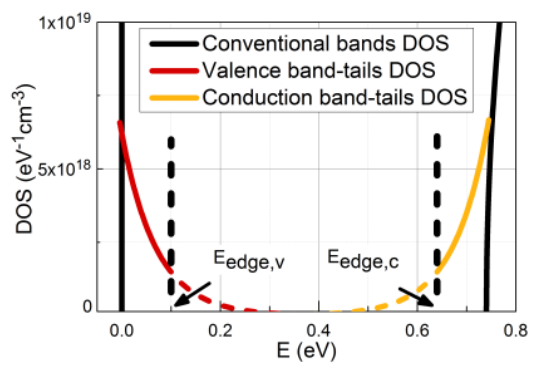

Fig. 3. Density of states (DOS) of the bulk valence and conduction band (black) together with the DOS of the valence (red) and conduction band-tails (gold). $E_{0, c / v}$, $k_{1, c / v}$ and $E_{1, c / v}$ are given in Table I.

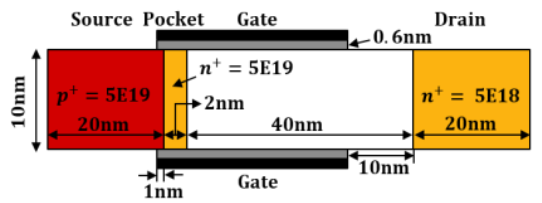

Fig. 6. Structure of the double-gate $p-n-i-n \mathrm{In}_{0.53} \mathrm{Ga}_{0.47}$ As tunnel-FET.

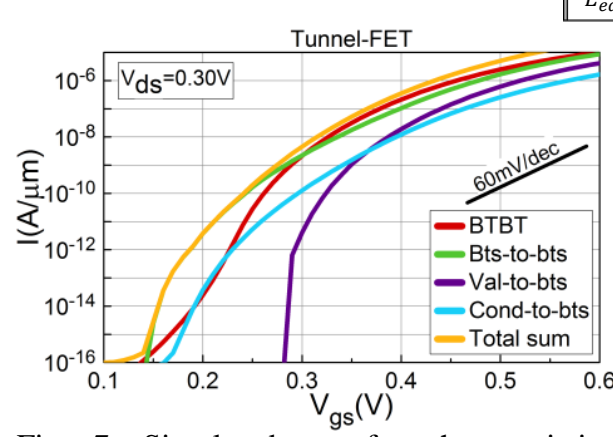

Fig. 7. Simulated transfer characteristics (gold) of the $p-n-i-n$ tunnel-FET structure of Fig. 6. Values used for $E_{0, c / v}, k_{1, c / v}, E_{1, c / v}$ and $E_{e d g e, c / v}$ are given in Table I.
TABLE I. CALIBRATED PARAMETERS VALUES.

\begin{tabular}{||l|c|}
\hline$E_{0, c}$ & $70 \mathrm{meV}$ \\
$k_{1, c}$ & $2.4 \times 10^{8} \mathrm{~m}^{-1}$ \\
$E_{1, c}$ & $E_{C}+5 \mathrm{meV}$ \\
$E_{\text {edge,c }}$ & $E_{C}-100 \mathrm{meV}$ \\
\hline$E_{0, v}$ & $70 \mathrm{meV}$ \\
$k_{1, v}$ & $2.4 \times 10^{8} \mathrm{~m}^{-1}$ \\
$E_{1, v}$ & $E_{V}-5 \mathrm{meV}$ \\
$E_{\text {edge }, v}$ & $E_{V}+100 \mathrm{meV}$ \\
\hline
\end{tabular}

